\title{
Linear Model of DHW System Using Response Surface Method Approach
}

\author{
Rajab GHABOUR*, Péter KORZENSZKY
}

\begin{abstract}
Using alternative and clean energy resources is considered the most effective method to deal with the environment and energy crises nowadays. In this study a comparative analysis of FPC data for DHW are theoretically and experimentally evaluated to optimise the design parameters. A forced circulation solar heating system using flat-plate collector, is modelled using T*SOL as a new approach, for hot water requirements of a laboratory unit at Szent Istvan University, in Gödöllö, Hungary. The modelling shows $69 \%$ solar fraction and $510 \mathrm{kWh}$ annual solar gain. Furthermore, the comparison study was based on these two factors, and the two results matched with $93.12 \%$ accuracy. After the modelling, practically, the exergy analysis was conducted to determine and highlight the losses of the system. Since Hungary is considered a cold climate country, it was found that the thermal and optical losses from the collector and the piping system were tremendous. Then all the necessary design parameters were studied to achieve the optimal working points using non-linear correlation response surface method (RSM, which has never been used as an analysis tool in the solar field) at two perspectives (solar fraction and annual yield) for seven factors using R script. The factors were collector surface area, inclination angle, glycol-water ratio, tank capacity, boiler capacity, desired hot water temperature, and volume flow-rate. We identified the most influential single factors using ParetoPlot and each two-factors' interaction using contour plots. The most influential factors on the solar yield are solar collector surface area, tank capacity, desired hot water temperature and volume flow rate by magnitude effects of $+129.7,+125.3,+50.9$ and $+17.2 \mathrm{kWh}$ per annum, respectively.
\end{abstract}

Keywords: heat process; modelling; solar thermal system; solar fraction

\section{INTRODUCTION}

Domestic hot water produced by the flat plate collector is considered the best opportunity to utilise solar energy, mainly in the building sector where $46 \%$ of the total worldwide demand is attributed to heating and cooling with more than $30 \%$ of greenhouse emissions in OEDD countries $[1,2]$. It has reached a maturity level, becoming the most developed solar system on the market implemented in many countries after the oil crisis in 1973 $[3,4]$. That made the share of renewable energy, globally, reach $10.4 \%$. Nevertheless, $79.6 \%$ is produced by conventional exploitation of fossil fuels [5]. Furthermore, to provide domestic hot water (DHW) renewable energy has recently become the centre of attention for its availability and low emission results [6]. As for space heating, solar systems are considered a good solution for a single-family house, where water is generally the medium used for both heat-storing and/or heat distribution. Different strategies are available and have been developed to deal with variable load profiles and heat sources. If the solar irradiation is not sufficient, an additional heat source such as a boiler run by gas or oil is needed to compensate for the surplus demand. An electrical element can also be integrated into the tank, which could exist in some systems.

The single-family load profile is characterised by a short duration of discharge $(<0.2$ hours) and a high heating rate of up to $50 \mathrm{~kW}[7,8]$. Usually, the system has the following main features: the water tank must maintain the main water at a sufficient temperature level to ensure satisfying the load, and the system has one or more immersed heat exchanger(s). The strongest influences on the energy savings are the hot water preparation, the thermostat controlling, and the load profile. Even though solar thermal energy is common nowadays, few DHW systems are intended for use with the solar heating system. Moreover, the load profile can be simplified for optimisation modelling [9], but in reality, the system performance could be non-optimal, which reflects the importance of this factor. On the other hand, the control sensor mounted at the bottom of the storage tank, where the temperature is lower than at the upper level, will extend the utilisation time of the solar energy [10]. Having a stable stratification by using a heat exchanger in the solar tank, can also lead to a 17.3 per cent higher utilised heat in the load side [10], and a 5.25 percent higher energy savings over a one year usage, compared to a fully mixed tank [3]. So thermal stratification is applied in different types of energy storage fields, like solar thermal systems, due to the sporadic characteristics of the solar radiation, making it an essential part of the long-term operation of the heating systems to minimise the mixing effect which happens due to the temperature difference between cold and hot water [11]. The optimum performance of the solar collector is proposed by the following: choosing a low conductivity material that allows reducing the weight and cost. Furthermore, the flow passageways are rectangular-shaped in order to increase the sturdiness.

The low flow in solar domestic hot water (SDHW) has several advantages, mainly in the fact that the outlet temperature will be higher from the solar collector which causes a higher degree of the thermal stratification in the storage tank, leading to a close match with the desired load temperature. Therefore, the solar fraction will be higher, and the auxiliary heating intervention will be less. Also, due to the high stratification in the storage tank, the return temperature to the solar collector will be less, so that the collector utilisation period will be extended. Moreover, the used pipes can be smaller, which means less material for pipes and insulation, and the heat loss will be lower because of the smaller-exposed area. Finally, the pump size can be reduced as well.

In terms of heat loss, the evacuated-tube collector has a significant advantage over the flat-plate collector, because of the weather variations and the sensitivity of each collector to those factors. So, the weather data is grouped into four categories: clear sky, hazy day, hazy and cloudy, and cloudy day where the diffusive radiation shares are $\leq 25 \%$, between $25-50 \%$, between $50-75 \%$, and $\geq 75 \%$ respectively [12]. Nevertheless, by analysing many existing facilities, it is shown that many of them have a dimensioning defect or are operated incorrectly [13]. This 
cannot just cause economic loss but also less satisfactory results in the overall gain [14]. Even though SDHW systems have a high reliability and satisfactory performance, it requires an adequate sizing and proper components' selection in order to accurately determine and predict the useful delivered energy and outlet water temperature [15]. The optimisation of the system parameters is crucial to achieving a good performance mainly in cold countries, where thermal losses from DHW systems are high, the solar irradiance is low, and the freezing of the system-medium is a big issue [16, 17]. Based on that, many efforts were conducted to determine the quantitative relations among the operating parameters. However, most of these studies had investigations over short periods and for the simplistic operating conditions. One proposal is to classify SDHW systems by the rate between annual hot water production over the needed auxiliary heat (litres/kWh) where higher value means better results and the best system is the one that produces more hot water per unit of auxiliary heating or in other words, the one that needs less auxiliary energy to produce one volume unit of hot water. In the previous research papers, the majority were focusing on warm regions and short-time analysis, where wintertime is mild, therefore no freezing happens in the medium. However, in cold regions where the outdoor temperature remains below the freezing point for a long time (about four to six months), the thermosyphon solar water heating system may not be an appropriate solution. However, using antifreeze and forced flow would be a suitable configuration for the solar system in cold countries. Besides, the flat-plate collectors are a cheap solution, easily fabricated and installed, and have low operating and maintenance costs compared to other collector types (ETC, CPC) [18].

Nowadays, many computational tools are being developed to evaluate numerically the solar system's long term performance. For instance, TRNSYS is a transient simulation software that both thermal and PV solar systems, renewable energy systems, and fuel cells can be modelled with. Most of the studies regarding solar systems have used this program to study the characteristics of horizontal and vertical tanks, tank height effects on the performance and the annual solar fraction, auxiliary heater position, thermal conductivity of the absorbing plate, and the optimal angle tilt of the collector. In all these researches, theoretical and experimental results are compared and found a good agreement. On the other hand, modelling with MATLAB became more common since it has several environment blocks such as SimSolar, which gives a highly accepted accuracy rate reaching up to 0.03 ${ }^{\circ} \mathrm{C}[19]$. On the one hand, $\mathrm{T}^{*} \mathrm{SOL}$ is a minute-by-minute dynamic simulation program for the calculation, optimisation, and design of solar thermal systems. It has the advantage of the parameters variation to identify the optimal working points, mainly for controlling processes such as switching temperatures and sensor heights, which has a major impact on efficiency. The present work is focused on $\mathrm{T}^{*} \mathrm{SOL}$ to analyse a forced circulation solar water heating system for a laboratory unit in Gödöllö, Hungary. This includes a comprehensive study of all the design parameters of the system to optimise its values, where monthly and annual solar fractions of the system are used as reference parameters to be optimised.
The gap found in the literature is that $\mathrm{T} * \mathrm{SOL}$ as a program was not as widely used compared to TRNSYS and MATLAB even though it has vast range of working parameters. On the other hand, the analysis and evaluation of the working system, if the many variables change at the same time, was never considered in the literature. So the core of our study will be to use a new approach for modelling and new analysis tool for multi-variable cases in a new set of seven major parameters in the solar DHW system.

\section{MATERIALS AND METHODS}

The process of the study is to be as in Fig. 1.

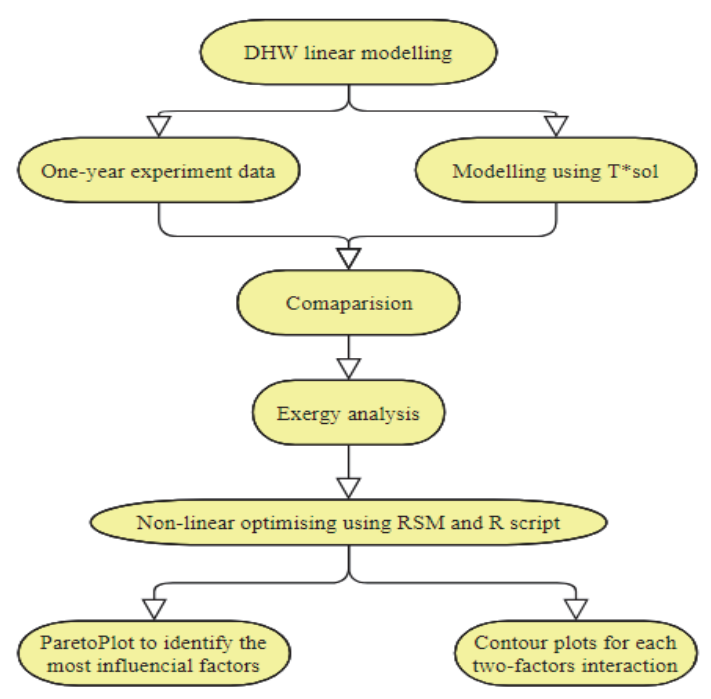

Figure 1 Flowchart of the study

The DHW system, as shown in Fig. 2a, is a direct forced circulation system with internal heat exchanger, consisting of a flat-plate solar collector mounted on the roof of the laboratory, a heat storage tank, and a supporting boiler. The primary flow, which transports and absorbs the solar energy, runs between the solar collector and the hot side of the heat exchanger. The flow medium is assumed to be a combination of the water-glycol mixture to avoid water freezing during wintertime, but in the studied realsystem case, it has a pure volume water mixture. This primary flow exchanges the energy between the cold water in the tank with the hot water in the solar collector. The energy is then transferred to the tank through the contact between the heat exchanger and the surrounding water. Before the final utilisation of the hot water, the controlling system compares the output water's temperature with the desired one (in our case $45^{\circ} \mathrm{C}$ ). If it matches, it can pass, otherwise, the parallel boiler will compensate for the needed energy in case of unsatisfactory solar energy gain (overcast days).

In contrast, when there is high solar radiation, the water may be warmer than the desired temperature. In this case, the water supply network mixes with the tank to achieve the desired temperature. An optional electrical heating element in the tank is considered the right solution, but in our case-study it does not exist. Our study focused on getting the closest simulation results compared to the real model, besides an optimisation methodology using 
response surface method (RSM) to optimise the factors. The simulation variables-set is listed in Tab. 1 with the most critical factors.

Table 1 Simulation variables of the real system

\begin{tabular}{|c|c|c|c|}
\hline Main group & Parameter & In reality & Unit \\
\hline \multirow{2}{*}{$\begin{array}{l}\text { Collector } \\
\text { array }\end{array}$} & $\begin{array}{l}\text { Active solar } \\
\text { surface }\end{array}$ & 1.92 & $\mathrm{~m}^{2}$ \\
\hline & Inclination & $33^{\circ}$ & \\
\hline \multirow{4}{*}{ Piping } & Length & $\begin{array}{c}\text { Inide } 3.7 \\
\text { Outside } 2.7 \\
\end{array}$ & $\begin{array}{l}\mathrm{m} \\
\mathrm{m}\end{array}$ \\
\hline & $\begin{array}{c}\text { Thermal } \\
\text { conductivity }\end{array}$ & PE 0.045 & $\mathrm{~W} / \mathrm{m} \cdot \mathrm{K}$ \\
\hline & Nominal diameter & 22 & $\mathrm{~mm}$ \\
\hline & $\begin{array}{c}\text { Insulation } \\
\text { thickness }\end{array}$ & $\begin{array}{c}\text { Inide } 36 \\
\text { Outside } 36\end{array}$ & $\begin{array}{l}\mathrm{mm} \\
\mathrm{mm}\end{array}$ \\
\hline \multirow{3}{*}{ Tank } & Volume & 471 & litres \\
\hline & $\begin{array}{l}\text { Insulation } \\
\text { thickness }\end{array}$ & 50 & $\mathrm{~mm}$ \\
\hline & $\begin{array}{c}\text { thermal } \\
\text { conductivity }\end{array}$ & 0.027 & $\mathrm{~W} / \mathrm{m} \cdot \mathrm{K}$ \\
\hline $\begin{array}{c}\text { Gas-fired } \\
\text { boiler }\end{array}$ & Nominal power & 21 & $\mathrm{~kW}$ \\
\hline \multirow{3}{*}{ DHW } & Consumption & 9957 & Litres/year \\
\hline & Temperature & $45^{\circ} \mathrm{C}$ & \\
\hline & Operating times & 6АM-5PM & Weekends off \\
\hline \multirow{3}{*}{$\begin{array}{l}\text { Collector } \\
\text { loop }\end{array}$} & Volume flow rate & $115 \mathrm{l} / \mathrm{h} / \mathrm{m}^{2}$ & $2401 / \mathrm{h}$ \\
\hline & Medium & $100 \%$ Water & $0 \%$ glycol \\
\hline & Control & on $+3 \mathrm{~K}$ off $-3 \mathrm{~K}$ & $\begin{array}{l}\text { Above tank } \\
\text { temperature }\end{array}$ \\
\hline
\end{tabular}

The main comparing parameter is the annual solar fraction, which stands for the total hot water supplied by the solar system compared to the total hot water demand as in the equation:

$$
f=\frac{Q_{\text {Load }}-Q_{\text {Auxiliary }}}{Q_{\text {Load }}}
$$

where $Q_{\text {Load }}$ is the total energy transferred from the system to heat the water and $Q_{\text {Auxiliary }}$ is the portion of the hot water that was heated by the auxiliary elements which could not be provided by the solar energy due to the weather conditions. The best indicator is the solar fraction since it illustrates the overall system performance compared to the collector efficiency of the heat removal factor, which manifests only one component, not an entire system. The collector efficiency is the monthly or annual ratio of the useful energy gain to the absorbed solar energy by the collector, which is computed from Duffie and Beckman [20]:

$$
\begin{aligned}
& \eta=\frac{Q_{\mathrm{u}}}{A_{\mathrm{c}} I_{\mathrm{T}}}=\frac{\dot{m} C_{\mathrm{p}}\left(T_{\text {out }}-T_{\text {in }}\right)}{A_{\mathrm{c}} I_{\mathrm{T}}}= \\
& =F_{\mathrm{R}}\left(\tau_{\alpha}\right)_{n}-F_{\mathrm{R}} U_{\mathrm{L}}\left(T_{\text {in }}-T_{\text {ambient }}\right)
\end{aligned}
$$

$F_{\mathrm{R}}$ is the heat removal factor, which stands for the actual useful energy gain to the useful gain if we assume that the entire plate is at the fluid inlet temperature from Duffie and Beckman [20]:

$$
F_{\mathrm{R}}=\frac{\dot{m} C_{\mathrm{P}}\left[1-\mathrm{e}^{-\frac{A_{\mathrm{C}} U_{\mathrm{L}} F^{\prime}}{\dot{m} C_{\mathrm{p}}}}\right]}{A_{\mathrm{c}} U_{\mathrm{L}}}
$$
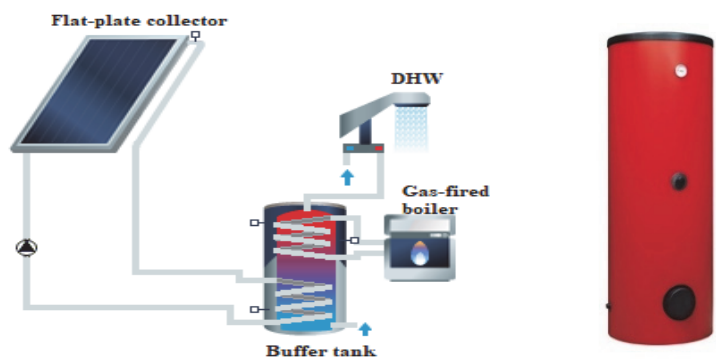

Figure 2 (a) Scheme of the direct DHW system; (b) Boiler tank ERR 500 litres

The tank is Hoval MultiVal ERR 500 litres made of robust steel with corrosion-resistant coating and highly effective thermal insulation causing low heat loss, with two internal heat exchangers (coils) to be used for heating using two heat sources. The primary heat source is usually a solar energy system, while the secondary one can be any type of heat generator, like a heat pump, wood pellet or a boiler. Optionally it can come with an electrical heating element. The tank is supported by a flange opening that can be used either for cleaning or for mounting the electrical heating element, as in Fig. $2 b$.

\section{RESULTS}

Firstly, we must insert the real-system parameters in $\mathrm{T} * \mathrm{SOL}$ and compare the annual solar contribution between the modelling $(510.6 \mathrm{kWh})$ and the real system $(477.7$ $\mathrm{kWh}$ ).

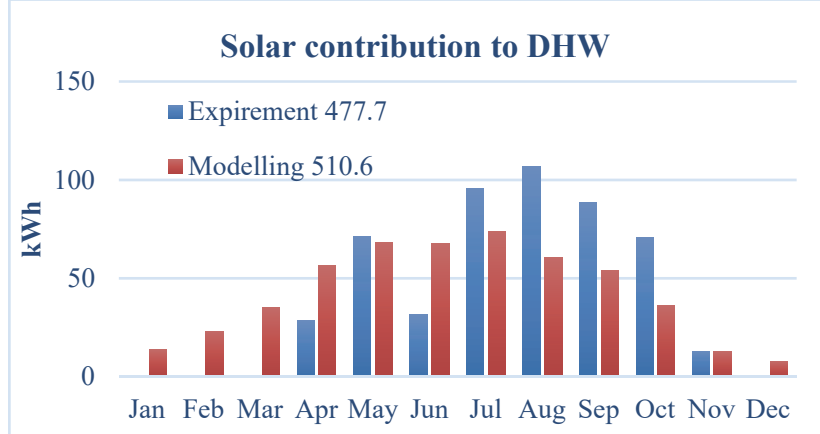

Figure 3 Solar contribution to DHW modelling and experiment

In Fig. 3, we can see that the difference in the annual gain is only $6.88 \%$, which is an accepted ratio. Nevertheless, there are some important notes about the results of the real experiment. The solar gain during June was low due to an unexpected rainy season so there is a slightly more significant mismatch between the two results in that month. Also, the control of the collector loop switches off at $-3 \mathrm{~K}$ below the tank's reference temperature, which is not accurate since it is logical to have it at least at $+1 \mathrm{~K}$, otherwise we are losing the energy from the tank, and this value is not accepted in the programming process. There may also be a deviation in the actual yields for the modelling values due to reasons such as climate fluctuations and consumption profile. That is why we see zero energy contribution from the experiment during Jan, Feb, Mar and Dec. Nevertheless, as shown, it has a low impact on the overall yield.

Without forgetting that our demand is relatively low, and the needed duty is for a laboratory, this system has a solar fraction of $69 \%$ and $19.1 \%$ efficiency, which, 
considered for this case, is a high ratio. The solar contribution as a percentage of the total consumption has a lesser degree during wintertime as in Fig. 4, where we can see that this system has an exemplary function from March untill October.

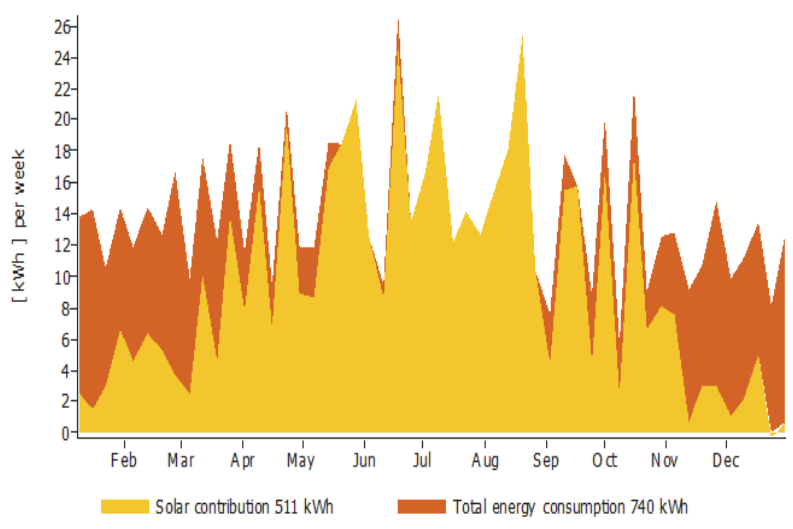

Figure 4 Solar contribution to DHW from total energy consumption

It is essential to have an exergy analysis (energy balance scheme) of the system to identify the losses and the gains as in Fig. 5. The optical and thermal losses, where optical losses represent the reflected solar radiation from the surface, while thermal losses represent the heat lost to the ambient, and both being important to estimate the total collector's efficicency, (in Fig. 5, legend 1.1 and 1.2) are 1575 from $2668 \mathrm{kWh}$ active irradiation on the collector surface which means that $60 \%$ of the achieved collector's energy is lost to the surroundings. Legends 2.5 and 2.6 also represent the internal and external piping losses of 100 and $272 \mathrm{kWh}$ respectively compared to $1093 \mathrm{kWh}$ passing the pipes, which means that the piping system has a $34 \%$ loss. Also, the boiler has an efficiency of $73.7 \%$, and finally, the tank has a $561 \mathrm{kWh}$ loss, as seen in legend 3.1, compared to 722 and 230 energy coming from the collector and the boiler as in legends 2.1 and 6.1 respectively which results in a $58.9 \%$ loss ratio. As we can see, the exergy analysis gives us factual information about the losses and how to overcome them.

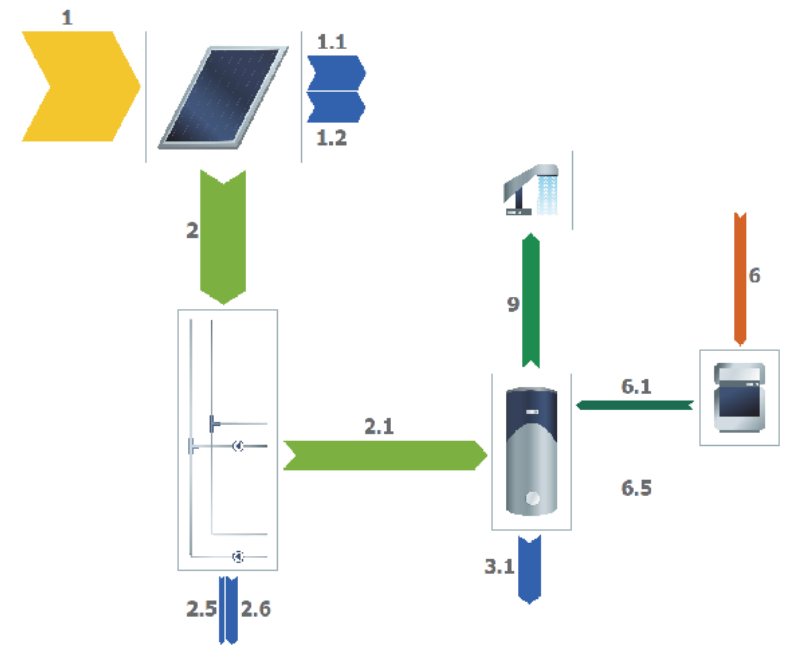

Figure $5 \mathrm{DHW}$ exergy analysis

Now we optimise the system, where the suggested model has $k=7$ factors. Each factor has two coded levels at -1 and +1 . Those coded values have a corresponding real value for each factor, as in Tab. 2, and to convert between real and coded values we use Eq. (4). If a full factorial experiment is conducted, the number of experiments should be $2^{k}$ which equals 128 experiments to optimise the model. However, as we increase the number of factors, we would have a lower resolution, higher aliasing, and extra costs. Therefore one-eights fractional factorial screening is conducted at three levels lower, meaning we have $2^{k-3}$ equaling 16 experiments, in addition to the real experiment of the system, as with number 17 in Tab. 3. The first screening has less information than the full experiment model, which reduces the knowledge of the system, and the estimation will not be highly accurate, but it is better to have a preliminary vision on the system before conducting the optimisation.

Table 2 Range of design parameters for the simulation

\begin{tabular}{|c|c|c|c|c|c|c|}
\hline Factor & $\begin{array}{c}\text { Design } \\
\text { parameters }\end{array}$ & $\begin{array}{c}\text { Low } \\
\text { level }\end{array}$ & \multicolumn{2}{|c|}{$\begin{array}{c}\text { Intermediary } \\
\text { levels }\end{array}$} & $\begin{array}{c}\text { High } \\
\text { level }\end{array}$ & Unit \\
\hline $\mathrm{A}$ & $\begin{array}{c}\text { Collector active } \\
\text { area }\end{array}$ & 0.5 & 1.66 & 2.83 & 4 & $\mathrm{~m}^{2}$ \\
\hline $\mathrm{B}$ & $\begin{array}{c}C_{\mathrm{P}} \text { (glycol-water) } \\
\text { ratio }\end{array}$ & 0 & 25 & 50 & 75 & $\%$ \\
\hline $\mathrm{C}$ & Inclination angle & 27 & 30 & 33 & 36 & ${ }^{0}$ \\
\hline $\mathrm{D}$ & Tank capacity & 100 & 300 & 500 & 700 & $\mathrm{Litres}$ \\
\hline $\mathrm{E}$ & Boiler capacity & 7 & 18 & 18.93 & 30 & $\mathrm{~kW}$ \\
\hline $\mathrm{F}$ & $\begin{array}{c}\text { Desired water } \\
\text { temperature }\end{array}$ & 40 & 49.6 & 50.4 & 60 & ${ }^{\circ} \mathrm{C}$ \\
\hline $\mathrm{G}$ & Volume flow rate & 20 & 125.9 & 134 & 240 & $1 / \mathrm{h} / \mathrm{m}^{2}$ \\
\hline
\end{tabular}

On the other hand, after conducting the screening of the fundamental factors, the optimisation can be deeper after knowing the most influential factors among those seven factors. It is essential to know that the resolution of the screening is IV design, which is useful for learning about the system, and understanding its characteristics. At the early stage we prefer to screen many factors, $k=7$, even though a very complex confounding pattern will appear, because we are uncertain of the magnitude of each factor's effect on the total system response. In the process of further optimisation, while we are approaching our optimum with the most influential factors, we will find the dominant two or three factors' interactions. By that, full factorial optimisation will have been conducted to investigate and estimate the magnitude effects where the intercept equals the following:

$$
\begin{aligned}
& I=A B C E=A B D F=A C D G= \\
& =C D E F=B D G E=B C F G=A E F G \\
& \text { codedvalue }=\frac{\text { realvalue }- \text { centervalue }}{\frac{1}{2} \text { range }}
\end{aligned}
$$

Here we will discuss only the factors that have a significant magnitude on the total system performance as in Fig. 6a and Fig. 7. The first interaction is between factors $B$ and $C$, which has a negative magnitude of -47.21 , meaning if the two factors have two different signs, one positive and the other one negative, the best performance of $C-B$ graph happens when the first factors are negative. The other one is positive so that the overall result will reverse into a positive magnitude on the solar yield. In the real-world values, it means either increasing the glycol 
ratio up to $70 \%$ (which is not practical because it will reduce the specific heat capacity of the primary loop) and decreasing the inclination to $27^{\circ}$. On the other hand, increasing the inclination to $36^{\circ}$ and reducing the glycol ratio is more logical because the solar angle suggested in the case of Budapest is $48^{\circ}$ based on annual calculations or $72^{\circ}$ based on winter case. With this logic, we are getting closer to the optimum angle and having the highest specific heat capacity of the medium, which means higher transferred energy from the primary loop.

Table 3 Experiment data

\begin{tabular}{|c|c|c|c|c|c|c|c|c|c|c|}
\hline No. & $A$ & $B$ & $C$ & $D$ & $E=A B C$ & $F=A B D$ & $G=A C D$ & $\begin{array}{c}y_{1 \%} \mathscr{g} \\
1 \%\end{array}$ & $\begin{array}{c}y \_\mathscr{Z} \\
/ \mathrm{kWh}\end{array}$ & $\begin{array}{l}y-\eta \\
1 \%\end{array}$ \\
\hline 1 & -1 & -1 & -1 & -1 & -1 & -1 & -1 & 26 & 111 & 15.9 \\
\hline 2 & +1 & -1 & -1 & -1 & +1 & +1 & +1 & 82 & 598 & 10.7 \\
\hline 3 & -1 & +1 & -1 & -1 & +1 & +1 & -1 & 27 & 143 & 21 \\
\hline 4 & +1 & +1 & -1 & -1 & -1 & -1 & +1 & 84 & 476 & 8.5 \\
\hline 5 & -1 & -1 & +1 & -1 & +1 & -1 & +1 & 26 & 113 & 16.1 \\
\hline 6 & +1 & -1 & +1 & -1 & -1 & +1 & -1 & 73 & 482 & 8.6 \\
\hline 7 & -1 & +1 & +1 & -1 & -1 & +1 & +1 & 25 & 135 & 19.2 \\
\hline 8 & +1 & +1 & +1 & -1 & +1 & -1 & -1 & 71 & 364 & 6.5 \\
\hline 9 & -1 & -1 & -1 & +1 & -1 & +1 & +1 & 27 & 174 & 25 \\
\hline 10 & +1 & -1 & -1 & +1 & +1 & -1 & -1 & 87 & 712 & 12.8 \\
\hline 11 & -1 & +1 & -1 & +1 & +1 & -1 & +1 & 27 & 152 & 22 \\
\hline 12 & +1 & +1 & -1 & +1 & -1 & +1 & -1 & 86 & 825 & 14.8 \\
\hline 13 & -1 & -1 & +1 & +1 & +1 & +1 & -1 & 36 & 239 & 34 \\
\hline 14 & +1 & -1 & +1 & +1 & -1 & -1 & +1 & 88 & 783 & 14 \\
\hline 15 & -1 & +1 & +1 & +1 & -1 & -1 & -1 & 38 & 221 & 32 \\
\hline 16 & +1 & +1 & +1 & +1 & +1 & +1 & +1 & 86 & 899 & 16 \\
\hline 17 & -0.086 & -1 & +0.333 & +0.237 & 0.029 & 0.02 & -0.007 & 70 & 557 & 21 \\
\hline 18 & $+1 / 3$ & $+1 / 3$ & $+1 / 3$ & $+1 / 3$ & $+1 / 27$ & $+1 / 27$ & $+1 / 27$ & 79 & 673 & 17.1 \\
\hline 19 & $-1 / 3$ & $-1 / 3$ & $-1 / 3$ & $-1 / 3$ & $-1 / 27$ & $-1 / 27$ & $-1 / 27$ & 70 & 481 & 21 \\
\hline
\end{tabular}

The second biggest two-factor interaction magnitude is $A$ and $E$ with a positive magnitude of +46.8 . To get a positive influence from this interaction, we must have either two negative or two positive signs so the sign of the two factors interaction will stay positive. The real-world meaning illustrates that by either increasing the surface area and the boiler capacity together, while the other solution is to reduce them both to near $(-1,-1)$ coded values which means $0.5 \mathrm{~m}^{2}$ and $7 \mathrm{~kW}$ boiler capacity. The true explanation beyond this is that the boiler, at even 7 $\mathrm{kW}$, is oversized and the suggestion from the software is 3 $\mathrm{kW}$, knowing that the annual demand is relatively low concerning our case.

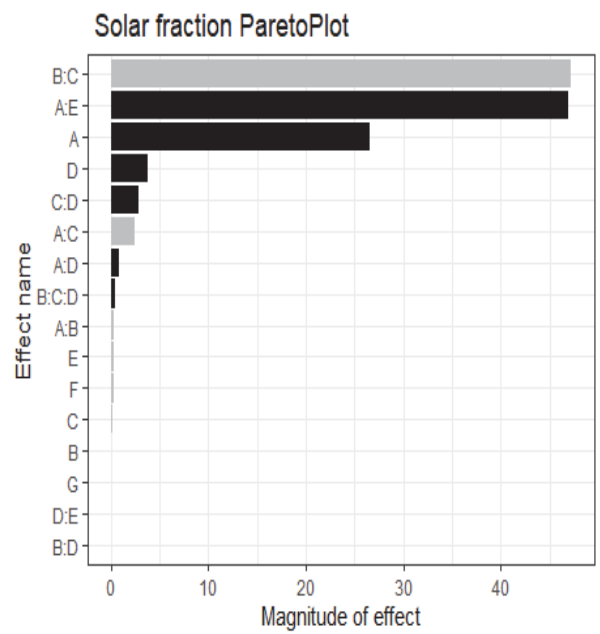

(a)

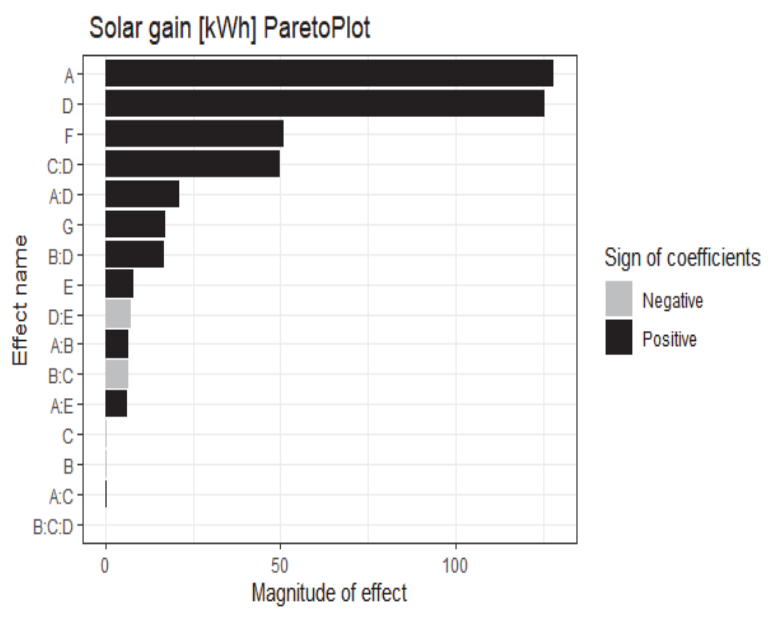

(b)

Figure 6 ParetoPlot (a) Solar fraction; (b) Solar gain

The next significant magnitude is the interaction between factors $C$ and $D$, which is +2.81 . From the contour, it is easy to note that the best design happens when both $C$ and $D$ are in a positive sign. In real-world values, it means that we increase the inclination angle and the tank capacity. If we draw a perpendicular line to the contour line, we note that to increase the overall performance we have to increase $D$ more than $C$. In real-world values it means that, to increase the tank capacity from 100 litres to 700 litres, has more influence than increasing the inclination angle from $27^{\circ}$ to $36^{\circ}$. Finally, as we saw in the screening process, factors $A$ and $D$ have the most significant single factor magnitude.

$$
\begin{aligned}
& y_{\mathcal{I} \%}=55.56+26.56 A-0.06 B-0.19 C+3.81 D-0.31 E-0.31 F+0.06 G-0.31 A^{*} B-2.44 A^{*} C-47.21 B^{*} C+ \\
& +0.81 A^{*} D-0.06 B^{*} D+2.81 C^{*} D+46.8 A^{*} E-0.06 D^{*} E+0.44 B^{*} C^{*} D \\
& y_{\mathcal{I} \mathrm{kWh}}=593.9+127.9 A-0.4 B-0.4 C+125.3 D+7.9 E+50.9 F+17.2 G+6.6 A^{*} B+0.3 A^{*} C-6.6 B^{*} C+ \\
& +21.1 A^{*} D+16.7 B^{*} D+49.7 C^{*} D+6.2 A^{*} E-7.4 D^{*} E+0.1 B^{*} C^{*} D
\end{aligned}
$$



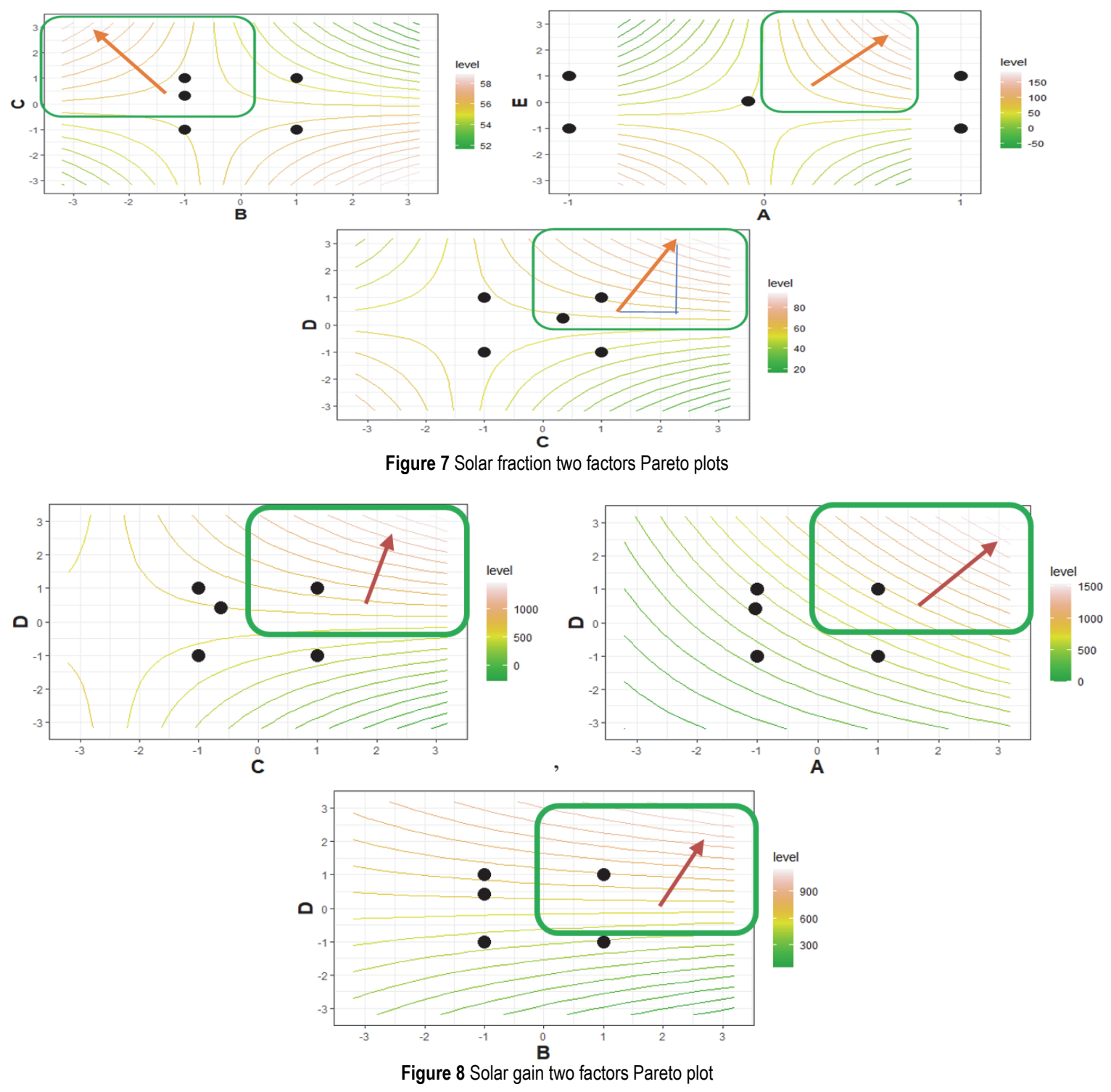

On the other hand, as in Fig. $6 \mathrm{~b}$ and Fig. 8, the twofactor interactions that significantly influence the solar gain are primarily $C$ and $D$ with 49.7 positive magnitude effect. From the contour plot, we can see that the highest yield happens when both factors are at higher coded values. This means higher tank capacity and, to a lesser degree, larger tilting angle as we can see from the slope of the contour lines. The next interaction is $A$ and $D$, which has a 21.1 positive magnitude effect. This states that we will get a higher solar yield when both $A$ and $D$ are in the same sign. The real meaning of this interaction is that the outcome will be higher if we choose a bigger collector area and a bigger tank capacity. Finally, there is the 16.7 positive magnitudes for the interaction between factor $B$ and $D$. From the contour lines we can distinguish, similarly to the $C-D$ interaction, that the better result will be a bigger tank capacity and, to a lesser degree, a hugher glycol ratio.

It is essential to ensure that the most influential single factors are the collector surface area and the tank capacity, which was distinguished and duplicated in the two ParetoPlots. Also, there is a single factor that has a substantial magnitude which was not observed in the solar fraction ParetoPlot, but was evident in the yield. Those factors are $G$ and $F$, which represent the desired hot water temperature and the volume flow rate. The reason behind it is that the desired hot water temperature is usually a specified input value, instead of a variable as in our case, where we can see some differences between the two ParetoPlots as well as in Tab. 3 at experiment 10 and 12 . We can see that experiment 12 has a higher solar fraction of $86 \%$ with $825 \mathrm{kWh}$ while experiment 10 has $87 \%$ solar fraction with $712 \mathrm{kWh}$. As we saw in the first experiment, the desired hot water was at $40^{\circ} \mathrm{C}$, while in the second one it was at $60{ }^{\circ} \mathrm{C}$. Those results have good agreement with the results of the reasrcher Hobbi, and Siddiqui who studied single factor magnitudes for cold region, in the case study of Montreal Canada.

We cannot read those results from a mathematical perspective only, for example, just to increase the area of the collector and the tank capacity, instead, we need to read the reason behind it. The duplication of the tank capacity and the collector surface area in both ParetoPlots, from another perspective, means that those two items have low efficiency, as we saw in the exergy analysis where we noticed that those two have a massive heat loss and need to be replaced by better quality components or to be improved retrofitly. While from a modelling aspect, $\mathrm{T} * \mathrm{SOL}$ should be compared with other tools in different climates to check its accuracy and limitations, and also optimised using methods other than RSM to make comparisons. 


\section{CONCLUSIONS}

A direct forced circulation solar DHW system with flat-plate collector providing hot water requirements for a laboratory entity in Gödöllö, Hungary, is modelled. The modelling had two steps; the first one was to achieve accurate results from $\mathrm{T} * \mathrm{SOL}$ at the similar real-system condition, and the second one to do optimisation of working parameters using $\mathrm{R}$ scripts and response surface method non-linear correlations (RSM). To facilitate our result readings, we generate ParetoPlots to identify each magnitude factor effect on the solar fraction and the annual solar yield. The modelling using $\mathrm{T}^{*} \mathrm{SOL}$ shows highly accurate results of the real system, and the matching ratio is $93.12 \%$. This modelling led us to have a deeper scope on this system using the exergy analysis to identify the losses from an energy balance schematic aspect. The result shows massive losses from the collector and the piping system in the outer part and the tank in the internal part of the system. This analysis used a practical perspective. Firstly, as in the exergy scheme, after that, from a mathematical perspective, where the RSM method showed each singlefactor and two-factor interaction. It was noted that the collector surface area and the tank capacity have a significant impact from the projection of the solar fraction. Besides, the desired hot water temperature and the volume flow-rate have a substantial influence on the solar yield.

\section{Acknowledgements}

This work was supported by the Stipendium Hungaricum Programme and by the Mechanical Engineering Doctoral School, Hungarian University of agriculture and life sciences, Gödöllö, Hungary.

\section{REFERENCES}

[1] Olsthoorn, D., Haghighat, F., \& Mirzaei, P. A. (2016). Integration of storage and renewable energy into district heating systems: a review of modelling and optimization. Solar Energy, 136. https://doi.org/10.1016/j.solener.2016.06.054

[2] Buonomano, A., Forzano, C., Kalogirou, S. A., \& Palombo, A. (2019). Building-façade integrated solar thermal collectors: Energy-economic performance and indoor comfort simulation model of a water based prototype for heating, cooling, and DHW production. Renewable Energy, 137, 20-36. https://doi.org/10.1016/j.renene.2018.01.059

[3] Cristofari, C., Notton, G., Poggi, P., \& Louche, A. (2003). Influence of the flow rate and the tank stratification degree on the performances of a solar flat-plate collector. International Journal of Thermal Sciences, 42(5), 455-469. https://doi.org/10.1016/S1290-0729(02)00046-7

[4] Ošlaj, M. \& Muršec, B. (2010). Biogas as a renewable energy source. Tehnički vjesnik, 17(1), 109-114.

[5] Bilardo, M., Fraisse, G., Pailha, M., \& Fabrizio, E. (2020). Design and experimental analysis of an Integral Collector Storage (ICS) prototype for DHW production. Applied Energy, 259(November 2019), 114104. https://doi.org/10.1016/j.apenergy.2019.114104

[6] Sabahi, H., Tofigh, A. A., Kakhki, I. M., \& Bungypoor-Fard, H. (2016). Design, construction and performance test of an efficient large-scale solar simulator for investigation of solar thermal collectors. Sustainable Energy Technologies and Assessments, 15, 35-41. https://doi.org/10.1016/j.seta.2016.03.004
[7] Bales, C. \& Persson, T. (2003). External DHW units for solar combi systems. Solar Energy, 74(3), 193-204. https://doi.org/10.1016/S0038-092X(03)00158-0

[8] Kolaković, I. \& Dović, D. (2009). Simulation of the solar domestic hot water system operation. Tehnički vjesnik, 16 (1), 3-9.

[9] Jordan, U. \& Vajen, K. (2001). Influence of the DHW load profile on the fractional energy savings: A case study of solar combi-system with TRNSYS simulations. Solar Energy, 69(prEN 12977), 197-208. https://doi.org/10.1016/S0038-092X(00)00154-7

[10] Géczy-Víg, P. \& Farkas, I. (2010). Neural network modelling of thermal stratification in a solar DHW storage. Solar Energy, 84(5), 801-806. https://doi.org/10.1016/j.solener.2010.02.003

[11] Han, Y. M., Wang, R. Z., \& Dai, Y. J. (2009). Thermal stratification within the water tank. Renewable and Sustainable Energy Reviews, 13(5), 1014-1026. https://doi.org/10.1016/j.rser.2008.03.001

[12] Mishra, R. K., Garg, V., \& Tiwari, G. N. (2015). Thermal modeling and development of characteristic equations of evacuated tubular collector (ETC). Solar Energy, 116, 165176. https://doi.org/10.1016/j.solener.2015.04.003

[13] de Keizer, A. C., Vajen, K., \& Jordan, U. (2011). Review of long-term fault detection approaches in solar thermal systems. Solar Energy, 85(7), 1430-1439. https://doi.org/10.1016/j.solener.2011.03.025

[14] Mezni, T., Zainine, M. A., Dakhlaoui, M. A., \& Zghal, A. (2017). Presentation and experimental validation of a solar DHW installation sizing control tool. International Journal of Hydrogen Energy, 42(48), 28958-28972. https://doi.org/10.1016/j.ijhydene.2017.04.129

[15] Hobbi, A. \& Siddiqui, K. (2009). Optimal design of a forced circulation solar water heating system for a residential unit in cold climate using TRNSYS. Solar Energy, 83(5), 700714. https://doi.org/10.1016/j.solener.2008.10.018

[16] Kumar, S. \& Mullick, S. C. (2010). Wind heat transfer coefficient in solar collectors in outdoor conditions. Solar Energy, 84(6), 956-963. https://doi.org/10.1016/j.solener.2010.03.003

[17] Januševičius, K., Bielskus, J., \& Martinaitis, V. (2019). Functionality assessment of building a microclimate system utilising solar energy in a cold climate. Journal of Mechanical Engineering, 65(4), 238-250. https://doi.org/10.5545/sv-jme.2018.5910

[18] Duomarco, J. L. (2015). Figure of merit for solar domestic hot water systems. Solar Energy, 111, 151-156. https://doi.org/10.1016/j.solener.2014.10.023

[19] Tóth, J. \& Farkas, I. (2019). Mathematical Modelling of Solar Thermal Collectors and Storages. Acta Technologica Agriculturae, 22(4), 128-133. https://doi.org/10.2478/ata-2019-0023

[20] Duffie, J. A. \& Beckman, W. A. (2013). Solar Engineering of Thermal Processes. John Wiley \& Sons, Inc. https://doi.org/10.1002/978111867160310pt

\section{Contact information}

Rajab GHABOUR, MSc

(Corresponding author)

Mechanical Engineering Doctoral School,

Hungarian University of Agriculture and Life Sciences,

Budapest, Hungary

E-mail: Ghabour.Rajab@stud.uni-mate.hu

Péter KORZENSZKY, habil. associate professor

Technical Institute,

Hungarian University of Agriculture and Life Sciences,

Budapest, Hungary

E-mail: Korzenszky.Peter.Emod@uni-mate.hu 